\title{
Resonant inelastic x-ray scattering study of $\mathrm{Ca}_{3} \mathrm{Ru}_{2} \mathrm{O}_{7}$
}

\author{
K. von Arx $\odot,{ }^{1},{ }^{*}$ F. Forte,,${ }^{2,3}$ M. Horio $\odot,{ }^{1}$ V. Granata $\odot,{ }^{3}$ Q. Wang $\odot,{ }^{1}$ L. Das, ${ }^{1}$ Y. Sassa,,${ }^{4}$ R. Fittipaldi, ${ }^{2,3}$ C. G. Fatuzzo, ${ }^{5,1, \dagger}$ \\ O. Ivashko $\odot,{ }^{1, \$}$ Y. Tseng, ${ }^{6}$ E. Paris, ${ }^{6}$ A. Vecchione,,${ }^{2,3}$ T. Schmitt, ${ }^{6}$ M. Cuoco $\odot,{ }^{2,3}$ and J. Chang $\odot^{1, \S}$ \\ ${ }^{1}$ Physik-Institut, Universität Zürich, Winterthurerstrasse 190, CH-8057 Zürich, Switzerland \\ ${ }^{2}$ CNR-SPIN, I-84084 Fisciano, Salerno, Italy \\ ${ }^{3}$ Dipartimento di Fisica “E.R. Caianiello”, Università di Salerno, I-84084 Fisciano, Salerno, Italy \\ ${ }^{4}$ Department of Physics, Chalmers University of Technology, SE-412 96 Gothenburg, Sweden \\ ${ }^{5}$ Institute of Physics, École Polytechnique Fedérale de Lausanne (EPFL), CH-1015 Lausanne, Switzerland \\ ${ }^{6}$ Swiss Light Source, Photon Science Division, Paul Scherrer Institut, CH-5232 Villigen PSI, Switzerland
}

(Received 27 April 2020; revised 5 August 2020; accepted 11 November 2020; published 1 December 2020)

\begin{abstract}
We present a combined oxygen $K$-edge x-ray absorption spectroscopy and resonant inelastic x-ray scattering (RIXS) study of the bilayer ruthenate $\mathrm{Ca}_{3} \mathrm{Ru}_{2} \mathrm{O}_{7}$. Our RIXS experiments on $\mathrm{Ca}_{3} \mathrm{Ru}_{2} \mathrm{O}_{7}$ were carried out on the overlapping planar and interplanar oxygen resonances, which are distinguishable from the apical one. Comparison to equivalent oxygen $K$-edge spectra recorded on band-Mott insulating $\mathrm{Ca}_{2} \mathrm{RuO}_{4}$ is made. In contrast to $\mathrm{Ca}_{2} \mathrm{RuO}_{4}$ spectra, which contain excitations linked to Mott physics, $\mathrm{Ca}_{3} \mathrm{Ru}_{2} \mathrm{O}_{7}$ spectra feature only intra- $t_{2 g}$ ones that do not directly involve the Coulomb energy scale. As found in $\mathrm{Ca}_{2} \mathrm{RuO}_{4}$, we resolve two intra- $t_{2 g}$ excitations in $\mathrm{Ca}_{3} \mathrm{Ru}_{2} \mathrm{O}_{7}$. Moreover, the lowest lying excitation in $\mathrm{Ca}_{3} \mathrm{Ru}_{2} \mathrm{O}_{7}$ shows a significant dispersion, revealing a collective character different from what is observed in $\mathrm{Ca}_{2} \mathrm{RuO}_{4}$. Theoretical modeling supports the interpretation of this lowest energy excitation in $\mathrm{Ca}_{3} \mathrm{Ru}_{2} \mathrm{O}_{7}$ as a magnetic transverse mode with multiparticle character, whereas the corresponding excitation in $\mathrm{Ca}_{2} \mathrm{RuO}_{4}$ is assigned to combined longitudinal and transverse spin modes. These fundamental differences are discussed in terms of the inequivalent magnetic ground-state manifestations in $\mathrm{Ca}_{2} \mathrm{RuO}_{4}$ and $\mathrm{Ca}_{3} \mathrm{Ru}_{2} \mathrm{O}_{7}$.
\end{abstract}

DOI: 10.1103/PhysRevB.102.235104

\section{INTRODUCTION}

Transition metal (TM) oxides with $4 d$ valence electrons often exhibit unconventional magnetic and electronic properties. These are dictated by the competition of comparable energy scales set by local interactions, including the Hund's rule and crystal field $(\mathrm{CF})$ terms, together with intrinsic spinorbit coupling (SOC) of TM ions. By entangling the electron spin to the shape of the electronic cloud in the crystal, SOC makes the electronic spin-orbital states highly sensitive to the intersite connectivity and effective dimensionality of the underlying lattice. One of the most important consequences is the possibility to tune the relative strength of competing magnetic interactions by varying the effective dimensionality in layered materials. Calcium-based ruthenates of the Ruddlesden-Popper family $\mathrm{Ca}_{n+1} \mathrm{Ru}_{n} \mathrm{O}_{3 n+1}$ offer one of the richest playgrounds with a great variety of phenomenology. The bilayer compound $\mathrm{Ca}_{3} \mathrm{Ru}_{2} \mathrm{O}_{7}$ and its derivatives have been the subject of intense investigations due to a multitude of interesting low-temperature properties, such as spin-valve and giant magnetoresistance effects [1-8]. It has been established

\footnotetext{
*karin.vonarx@uzh.ch

†Present address: Istituto di Scienze del Patrimonio Culturale, Consiglio Nazionale delle Ricerche (ISPC-CNR), Via Biblioteca 4, 95124 Catania, Italy.

ॠPresent address: Deutsches Elektronen-Synchrotron DESY 22607 Hamburg, Germany.

§johan.chang@physik.uzh.ch
}

that $\mathrm{Ca}_{3} \mathrm{Ru}_{2} \mathrm{O}_{7}$ undergoes a magnetic transition at $T_{N}=56 \mathrm{~K}$ and an electronic transition at $T_{s}=48 \mathrm{~K}$ [9-11]. The latter transition is-due to a steep uprise of the out-of-plane resistivity $\rho_{c}$ [4] — sometimes referred to as a metal-insulator transition even though the ground state is semimetallic $[10,11]$. Another reason is the lattice response across $T_{s}$. Cooling below $T_{s}$ generates a $c$-axis lattice parameter compression and, through the Poisson's relation, an in-plane lattice parameter enhancement [12]. This resembles what happens at the metal-insulator transition $(350 \mathrm{~K})$ of $\mathrm{Ca}_{2} \mathrm{RuO}_{4}[13,14]$. There, the $c$-axis compression leads to an almost fully occupied $d_{x y}$ orbital and a Mott-gap opening in the half-filled $d_{x z}$ and $d_{y z}$ bands. However, the effect in $\mathrm{Ca}_{3} \mathrm{Ru}_{2} \mathrm{O}_{7}$ is much smaller $(0.1 \%$ and $>1 \%$ compression of the lattice parameter $c$ in $\mathrm{Ca}_{3} \mathrm{Ru}_{2} \mathrm{O}_{7}$ and $\mathrm{Ca}_{2} \mathrm{RuO}_{4}$, respectively) $[12,15]$. The fact that both $\mathrm{Ca}_{3} \mathrm{Ru}_{2} \mathrm{O}_{7}$ and $\mathrm{Ca}_{2} \mathrm{RuO}_{4}$ undergo similar $c$ axis compressive transitions but end up with different ground states makes comparative studies interesting. In addition to the electronic properties, the magnetic ground states of these two compounds differ as well. Whereas $\mathrm{Ca}_{2} \mathrm{RuO}_{4}$ displays a G-type antiferromagnetic state below $T_{N}=110 \mathrm{~K}$ [16], the in-plane magnetic moments in $\mathrm{Ca}_{3} \mathrm{Ru}_{2} \mathrm{O}_{7}$ order ferromagnetically leading to an A-type antiferromagnetic state [12]. This difference in the in-plane magnetic order implies that the interaction within the layers plays an important role for the magnetic ground state of these compounds. The investigation of the magnetic and orbital degrees of freedom and their excitation spectrum therefore offers a view on the complex interplay between different energy scales relevant for the ground state. In this respect, recent spectroscopic and neutron 

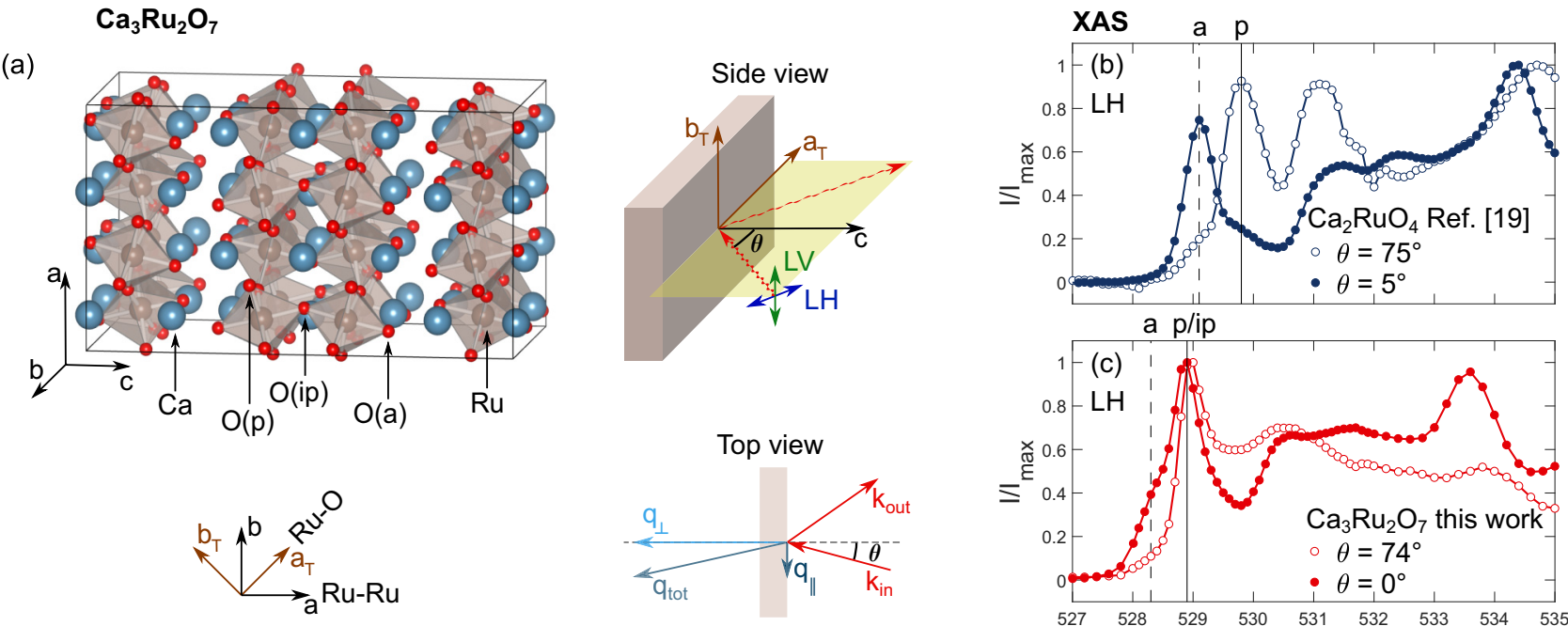

a $\mathrm{p} / \mathrm{ip}$
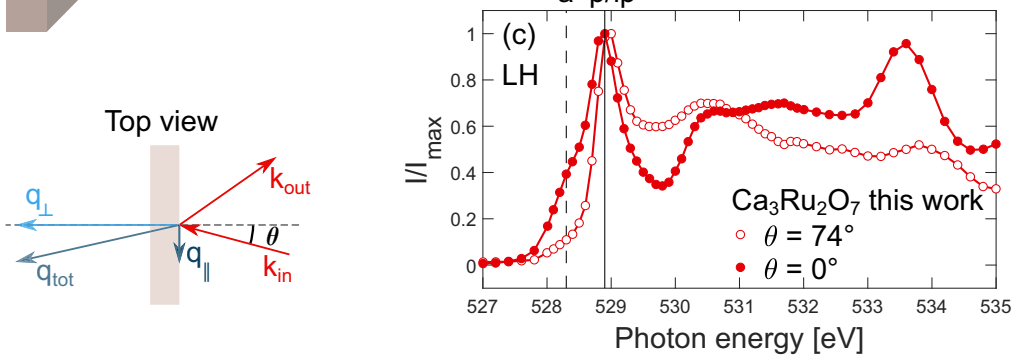

FIG. 1. (a) Schematically depicted RIXS geometry with respect to the crystal lattice of $\mathrm{Ca}_{3} \mathrm{Ru}_{2} \mathrm{O}_{7}$. Different oxygen sites in the crystal structure are labeled as $\mathrm{O}(\mathrm{p}), \mathrm{O}(\mathrm{a})$, and $\mathrm{O}(\mathrm{ip})$ for planar, apical, and interplanar sites, respectively. Momentum dependence was measured along the Ru-O direction. Therefore, the reciprocal space is indexed in tetragonal notation with $a_{T} \approx b_{T} \approx 1 / 2 \sqrt{a^{2}+b^{2}}$, where $a=5.37 \AA$ and $b=5.54 \AA$ [12]. (b), (c) Background subtracted and normalized XAS spectra of $\mathrm{Ca}_{2} \mathrm{RuO}_{4}$ and $\mathrm{Ca}_{3} \mathrm{Ru}_{2} \mathrm{O}_{7}$, respectively. The spectra were recorded with LH polarization near grazing and normal incident light directions as indicated. The dashed vertical black line indicates apical, whereas the solid vertical black line indicates planar $\left(\mathrm{Ca}_{2} \mathrm{RuO}_{4}\right)$ and overlapping planar and interplanar $\left(\mathrm{Ca}_{3} \mathrm{Ru}_{2} \mathrm{O}_{7}\right)$ oxygen resonances probing $\mathrm{Ru} t_{2 g}$ states. Dark blue data on $\mathrm{Ca}_{2} \mathrm{RuO}_{4}$ are taken from Ref. [19].

scattering measurements demonstrated that the magnetic ordering in $\mathrm{Ca}_{2} \mathrm{RuO}_{4}$ may sustain both longitudinal and transverse magnon modes with a large anisotropy gap, which reflects the impact of broken tetragonal symmetry in combination with SOC [17-19].

In this paper, we present a combined oxygen $K$-edge x-ray absorption spectroscopy (XAS) and resonant inelastic x-ray scattering (RIXS) study of $\mathrm{Ca}_{3} \mathrm{Ru}_{2} \mathrm{O}_{7}$ and compare it to previously published work on $\mathrm{Ca}_{2} \mathrm{RuO}_{4}$ [19,20], with the aim to investigate the distinctive fingerprints of the magnetic state in the single and bilayer compounds. With this methodology, the $\mathrm{Ru} 4 d$ orbitals are accessed indirectly through their hybridization with oxygen $p$ orbitals. In this fashion, we probe the two unoccupied $t_{2 g}$ states. This indirect approach has routinely been applied to different TM oxides [21-24].

Our study demonstrates that, in $\mathrm{Ca}_{3} \mathrm{Ru}_{2} \mathrm{O}_{7}$, only the two lowest intra- $t_{2 g}$ excitations are observed, whereas in $\mathrm{Ca}_{2} \mathrm{RuO}_{4}$, the Mott insulating ground state produces a set of excitations within the $t_{2 g}$ subspace, which consists of two low-energy and two mid/high-energy structures. An important differencethe main observation reported here-is that the lowest lying excitation exhibits a clear dispersive character in $\mathrm{Ca}_{3} \mathrm{Ru}_{2} \mathrm{O}_{7}$. This marked collective behavior is not found with the corresponding excitation in $\mathrm{Ca}_{2} \mathrm{RuO}_{4}$. The fundamentally different magnetic ground states of $\mathrm{Ca}_{2} \mathrm{RuO}_{4}$ and $\mathrm{Ca}_{3} \mathrm{Ru}_{2} \mathrm{O}_{7}$ are therefore manifested in the excitation spectrum, both within the $t_{2 g}$ and between the $t_{2 g}$ and $e_{g}$ sectors. We discuss this within the theoretical framework of fast collision approximation for the RIXS cross section $[25,26]$. Taking into account the different magnetic ground states of $\mathrm{Ca}_{2} \mathrm{RuO}_{4}$ and $\mathrm{Ca}_{3} \mathrm{Ru}_{2} \mathrm{O}_{7}$, qualitative agreement between calculated and observed RIXS spectra is obtained. The model qualitatively describes the marked differences in the RIXS spectra recorded on $\mathrm{Ca}_{2} \mathrm{RuO}_{4}$ and
$\mathrm{Ca}_{3} \mathrm{Ru}_{2} \mathrm{O}_{7}$. Moreover, we analyze the nature of the lowest lying intra- $t_{2 g}$ excitation. In this fashion, we show that this excitation is magnetic in both compounds, but with fundamentally different natures. In $\mathrm{Ca}_{2} \mathrm{RuO}_{4}$, the lowest lying excitation is consistent with composite longitudinal amplitude and transverse spin modes, whereas in $\mathrm{Ca}_{3} \mathrm{Ru}_{2} \mathrm{O}_{7}$ it has a dominant transverse spin nature.

These results provide decisive evidence for the capability of oxygen $K$-edge RIXS in probing the complex structure of electronic excitations in $4 d$ ruthenates. Particularly, it is confirmed that the low-energy spin/orbital modes are also directly accessible in virtue of modest SOC [23]. Such elementary excitations reflect the balance among competing interactions, being therefore crucial for revealing the origin of emergent phases and for determining the low-energy Hamiltonian in layered ruthenates, where magnetic interactions are no longer dictated by a global spin SU(2) symmetry alone.

\section{METHODS}

High quality single crystals of $\mathrm{Ca}_{3} \mathrm{Ru}_{2} \mathrm{O}_{7}$ were grown by the floating zone techniques [27,28], aligned ex situ by x-ray Laue and cleaved in situ using the top-post method. XAS and RIXS [26] measurements were carried out at the ADRESS beamline $[29,30]$ of the Swiss Light Source (SLS) at the Paul Scherrer Institut. The scattering geometry is indicated in Fig. 1(a). A fixed angle of $130^{\circ}$ between incident and scattered light was used. In-plane momentum $\mathbf{q}_{\|}=(h 2 \pi / a, k 2 \pi / b)$ is varied by controlling the incident photon angle $\theta$. In this work, the reciprocal space is indexed in tetragonal notation. Grazing and normal incidence conditions refer to $\theta \approx 90^{\circ}$ and $0^{\circ}$, respectively. Linear vertical (LV) and horizontal (LH) light polarizations were used to probe the oxygen $K$ edge at 

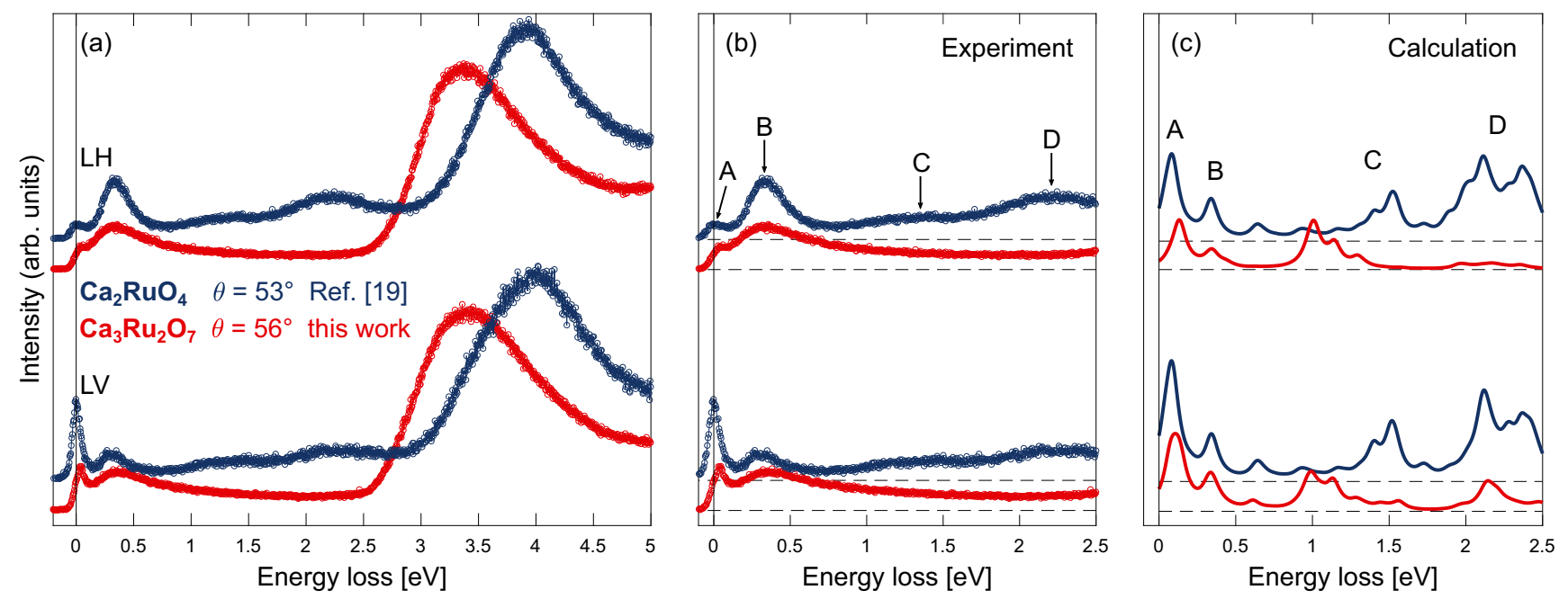

FIG. 2. (a) RIXS spectra of $\mathrm{Ca}_{2} \mathrm{RuO}_{4}$ and $\mathrm{Ca}_{3} \mathrm{Ru}_{2} \mathrm{O}_{7}$ recorded with $\mathrm{LH}$ and $\mathrm{LV}$ polarization as indicated. To enhance visibility, the spectra are given an individual vertical shift. Dark blue data on $\mathrm{Ca}_{2} \mathrm{RuO}_{4}$ are taken from Ref. [19]. (b) Zoom of (a) to show the low-energy excitations labeled as A, B, C, and D. (c) Calculated RIXS spectra of $\mathrm{Ca}_{2} \mathrm{RuO}_{4}$ and $\mathrm{Ca}_{3} \mathrm{Ru}_{2} \mathrm{O}_{7}$ for $\mathrm{LH}$ and LV polarization.

which an energy resolution of $22.5 \mathrm{meV}$ (Gaussian standard deviation $\sigma$ ) on $\mathrm{Ca}_{3} \mathrm{Ru}_{2} \mathrm{O}_{7}$ spectra was obtained. Elastic scattering is modeled by a Gaussian line shape (see Appendix C for details) with $\sigma$ set by the energy resolution. The presented data on $\mathrm{Ca}_{3} \mathrm{Ru}_{2} \mathrm{O}_{7}$ is collected at the base temperature $T=$ $20 \mathrm{~K}$ unless otherwise indicated. Our experimental setup for XAS and RIXS measurements on $\mathrm{Ca}_{3} \mathrm{Ru}_{2} \mathrm{O}_{7}$ is equivalent to that previously used for measurements of $\mathrm{Ca}_{2} \mathrm{RuO}_{4}$. Additionally, the energy resolution is comparable in both experiments and the minor base temperature differences (16-20 K) are negligible compared to the magnetic and electronic transitions in the two systems. Therefore, results on the two compounds are directly comparable.

\section{RESULTS}

The oxygen $K$-edge XAS spectra taken with LH light polarization on $\mathrm{Ca}_{2} \mathrm{RuO}_{4}$ and $\mathrm{Ca}_{3} \mathrm{Ru}_{2} \mathrm{O}_{7}$ are shown in Figs. 1(b) and 1(c). For $\mathrm{Ca}_{2} \mathrm{RuO}_{4}$, the apical and planar oxygen resonances are disentangled by using LH light near normal or grazing conditions, respectively [19,20]. For tetragonal and their orthorhombic derivatives, the crystal field (chemical) environment [22,31,32] and Coulomb interaction impose the apical oxygen edge to appear at lower photon energy than the planar oxygen edge. On this basis, the first and second pre-edges, indicated by dashed and solid vertical lines, correspond respectively to the resonances at the apical and planar oxygen sites, from which hybridization with $\mathrm{Ru} t_{2 g}$ orbitals takes place. In the case of $\mathrm{Ca}_{3} \mathrm{Ru}_{2} \mathrm{O}_{7}$, there are three oxygen sites: planar $\mathrm{O}(\mathrm{p})$, interplanar $\mathrm{O}(\mathrm{ip})$, and apical $\mathrm{O}(\mathrm{a})$ see Fig. 1(a). Compared to $\mathrm{Ca}_{2} \mathrm{RuO}_{4}$, these oxygen sites are harder to distinguish in the XAS spectra of $\mathrm{Ca}_{3} \mathrm{Ru}_{2} \mathrm{O}_{7}$ as the planar $\mathrm{O}(\mathrm{p})$ and interplanar $\mathrm{O}(\mathrm{ip})$ sites have similar $\mathrm{CF}$ environments. Similar to other layered oxides [22,31], we assign the first pre-edge of $\mathrm{Ca}_{3} \mathrm{Ru}_{2} \mathrm{O}_{7}$ to the $\mathrm{O}$ (a) site. In the normal $\left(\theta=0^{\circ}\right)$ condition, this resonance appears as a shoulder $(528.3 \mathrm{eV})$ on the second pre-edge $(528.9 \mathrm{eV})$ that is assigned to the $\mathrm{O}(\mathrm{p})$ and $\mathrm{O}(\mathrm{ip})$ sites. The reduced splitting of the oxygen $K$ pre-edges is also known from the XAS study of the Ruddlesden-Popper $\mathrm{Sr}_{1+n} \mathrm{Ru}_{n} \mathrm{O}_{3 n+1}$ series with $n=1,2$, and 3 [33,34]. Eventually, for cubic $\mathrm{SrRuO}_{3}$, the two pre-edges merge together and only one feature is observed [35]. The features at higher energies correspond to resonances probing the $\mathrm{O} p$ orbitals hybridized with the unoccupied $\mathrm{Ru} e_{g}$ states.

For our RIXS study of $\mathrm{Ca}_{3} \mathrm{Ru}_{2} \mathrm{O}_{7}$, we have focused entirely on the most intense oxygen $K$ pre-edge $(528.9 \mathrm{eV})$ that probes the planar and interplanar sites. In Figs. 2(a) and 2(b), spectra recorded with LV and LH light are compared to the corresponding planar spectra of $\mathrm{Ca}_{2} \mathrm{RuO}_{4}$. First, we notice that the "block" of $d d$ excitations in $\mathrm{Ca}_{3} \mathrm{Ru}_{2} \mathrm{O}_{7}$ around $3.5 \mathrm{eV}$ is consistently shifted to lower energies relative to what is found in $\mathrm{Ca}_{2} \mathrm{RuO}_{4}$. Another noticeable difference is that among the four "low" energy excitations reported [19] for $\mathrm{Ca}_{2} \mathrm{RuO}_{4}$ [labeled as A, B, C, and D in Fig. 2(b)], only the two lowest ( $\mathrm{A}$ and $\mathrm{B}$ ) are found in $\mathrm{Ca}_{3} \mathrm{Ru}_{2} \mathrm{O}_{7}$. The $\mathrm{B}$ excitation of bilayer $\mathrm{Ca}_{3} \mathrm{Ru}_{2} \mathrm{O}_{7}$ has a significantly smaller amplitude and is much broader than in $\mathrm{Ca}_{2} \mathrm{RuO}_{4}$. However, for both compounds, the $\mathrm{B}$ excitation is more intense when probed with LH polarization; see Fig. 3(a).

The lowest lying excitation (labeled as A) is overlapping with the elastic line and careful analysis is required to separate these two contributions. Elastic scattering is most pronounced near the specular condition; therefore, the A excitation appears as a shoulder on the energy loss side-see Fig. 3(b). Near grazing condition, the situation is reversed and the elastic scattering appears as a shoulder on the left side of the A excitation peak. To model the elastic contribution, we use a Gaussian profile with the linewidth set by the energy resolution. In this fashion, it is possible to extract the A excitation by subtracting the elastic component as well as the contributions from the B excitation and background, as illustrated in Figs. 3(b) and 3(c). As the incidence angle-and hence the in-plane momentum transfer-is varied, the A excitation is dispersing to a lower energy away from the zone center. Finally, the A excitation persists at least up to $80 \mathrm{~K}$, as shown in Fig. 3(c). 

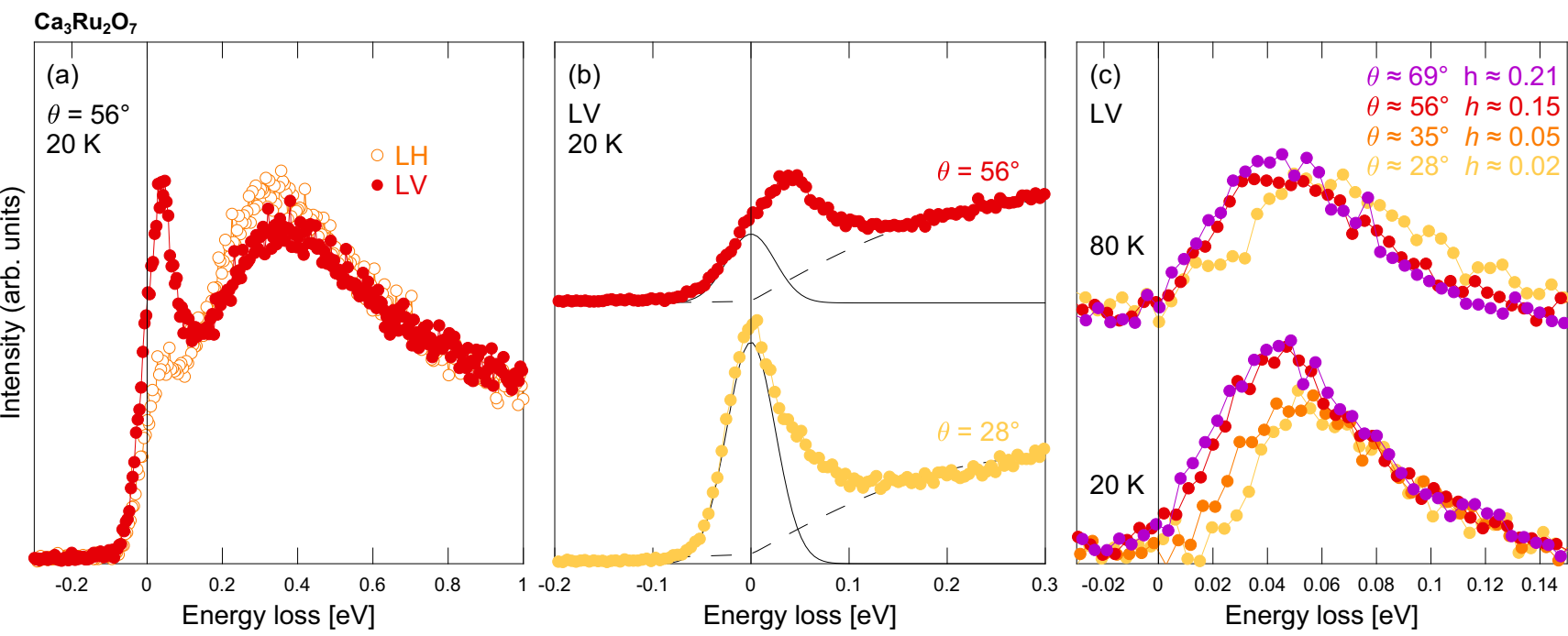

FIG. 3. RIXS spectra of $\mathrm{Ca}_{3} \mathrm{Ru}_{2} \mathrm{O}_{7}$ focusing on the low-energy part. (a) Comparison between LH and LV spectra at the same incidence angle. (b) Low-energy part for two different incidence angles measured with LV polarization. Solid black lines are Gaussian fits with a width fixed to the energy resolution of the experiment to model the elastic scattering. Dashed black lines are the sums of B excitation and background contributions. To enhance visibility, the spectrum at higher angle is given a vertical shift. (c) RIXS spectra after subtraction of the elastic line, B excitation, and background contributions to show the dispersion of the A excitation at $20 \mathrm{~K}$ and $80 \mathrm{~K}$, as indicated. To enhance visibility, the spectra at $80 \mathrm{~K}$ are given a vertical shift. The indicated $\theta$ and $h$ values correspond to the $20 \mathrm{~K}$ data; values for $80 \mathrm{~K}$ data differ slightly-see Fig. 4.

The momentum dependence of the $\mathrm{A}$ and $\mathrm{B}$ excitations extracted from the $\mathrm{Ca}_{3} \mathrm{Ru}_{2} \mathrm{O}_{7}$ data are compared in Fig. 4 to the corresponding excitations in $\mathrm{Ca}_{2} \mathrm{RuO}_{4}$. For the $\mathrm{B}$ excitation, the peak position is defined as the maximum obtained from the derivative of the spectrum, since the peak is extremely broad. Within the energy resolution of this experiment, no momentum dependence can be resolved for this excitation in $\mathrm{Ca}_{3} \mathrm{Ru}_{2} \mathrm{O}_{7}$. The situation is different in $\mathrm{Ca}_{2} \mathrm{RuO}_{4}$, where a small upward dispersion away from the zone center is detected for the B excitation [19]. Most pronounced differences are observed for the A excitation. The strong dispersion found in $\mathrm{Ca}_{3} \mathrm{Ru}_{2} \mathrm{O}_{7}$ is completely absent in $\mathrm{Ca}_{2} \mathrm{RuO}_{4}$. Additionally, the excitation is located at significantly higher energies in $\mathrm{Ca}_{2} \mathrm{RuO}_{4}$ at around $80 \mathrm{meV}$ compared to $55 \mathrm{meV}$ in $\mathrm{Ca}_{3} \mathrm{Ru}_{2} \mathrm{O}_{7}$. We stress that the A excitation dispersion is measured with LV polarization and hence is probed on either the planar or interplanar site via the $p_{y}$ orbital independent of $\theta$. The observed dispersion can therefore not be assigned to scattering geometry effects.

\section{DISCUSSION}

To discuss the XAS spectra, we first summarize the interpretation of the $\mathrm{Ca}_{2} \mathrm{RuO}_{4}$ data published recently [19,20]. The exact mechanism behind the Mott insulating state of $\mathrm{Ca}_{2} \mathrm{RuO}_{4}$ has long been under discussion and various theoretical models have been proposed [36-38]. In this context, the $\mathrm{Ca}_{2} \mathrm{RuO}_{4} \mathrm{XAS}$ results strongly support the explanation via a complete orbital polarization with the almost fully occupied $d_{x y}$ orbital. Indeed, the XAS spectra, reproduced in Fig. 1(b), are in perfect accordance with this picture. As discussed in Ref. [19], the dominant XAS response flips from the apical to the planar resonance when changing from normal to near grazing incidence using LH polarized light. This geometry effect is a result of the almost fully occupied Ru $d_{x y}$ orbital, that is unavailable for absorption. Comparing the $\mathrm{Ca}_{2} \mathrm{RuO}_{4}$ and $\mathrm{Ca}_{3} \mathrm{Ru}_{2} \mathrm{O}_{7}$ spectra, the differences in crystal structure and orbital occupation become apparent. Due to the nonequivalent apical oxygen sites in $\mathrm{Ca}_{3} \mathrm{Ru}_{2} \mathrm{O}_{7}$, the apical feature splits and the outer apical $\mathrm{O}(\mathrm{a})$ is only visible as a shoulder to the strong planar resonance that overlaps with the interplanar $\mathrm{O}$ (ip) resonance. Taking into account the relative intensities of the two features, the XAS results suggest a different orbital occupation than in $\mathrm{Ca}_{2} \mathrm{RuO}_{4}$, with an only partially filled $d_{x y}$. This partial occupation is also in accordance with the reduced $c$-axis compression in $\mathrm{Ca}_{3} \mathrm{Ru}_{2} \mathrm{O}_{7}$ compared to $\mathrm{Ca}_{2} \mathrm{RuO}_{4}[12,15]$.

Next, we turn to discuss the RIXS spectra. The fact that completely different oxygen $K$-edge RIXS spectra are observed for $\mathrm{Ca}_{2} \mathrm{RuO}_{4}$ and $\mathrm{Ca}_{3} \mathrm{Ru}_{2} \mathrm{O}_{7}$ is a beautiful example of how ground state fingerprints are encoded into the excitations. In principle, the $\mathrm{CF}$ environment around an in-plane oxygen should be similar for $\mathrm{Ca}_{2} \mathrm{RuO}_{4}$ and $\mathrm{Ca}_{3} \mathrm{Ru}_{2} \mathrm{O}_{7}$. Yet, the RIXS excitation spectra are fundamentally different for these two compounds. In $\mathrm{Ca}_{2} \mathrm{RuO}_{4}$, a sequence of excitations has been identified in the $t_{2 g}$ sector, which are separated from the higher energy $t_{2 g} \rightarrow e_{g}$ features in the energy range $\sim 3-5$ eV $[19,40]$ - see Fig. 2(a). In particular, two broad excitations located around $1 \mathrm{eV}$ and $2 \mathrm{eV}$, labeled as $\mathrm{C}$ and $\mathrm{D}$, are linked to the energy scales of Hund's coupling and Coulomb interaction responsible for the Mott insulating ground state. In semimetallic $\mathrm{Ca}_{3} \mathrm{Ru}_{2} \mathrm{O}_{7}$ by contrast, these excitations are completely absent-see Fig. 2(b). Even within the lower energy $t_{2 g}$ sector, pronounced differences are identified. Although two excitations (labeled as A and B) -with similar energy scales-are resolved for both compounds, they appear to have a fundamentally different nature. $\mathrm{In} \mathrm{Ca}_{3} \mathrm{Ru}_{2} \mathrm{O}_{7}$ the lowest lying excitation is clearly dispersive, whereas in 

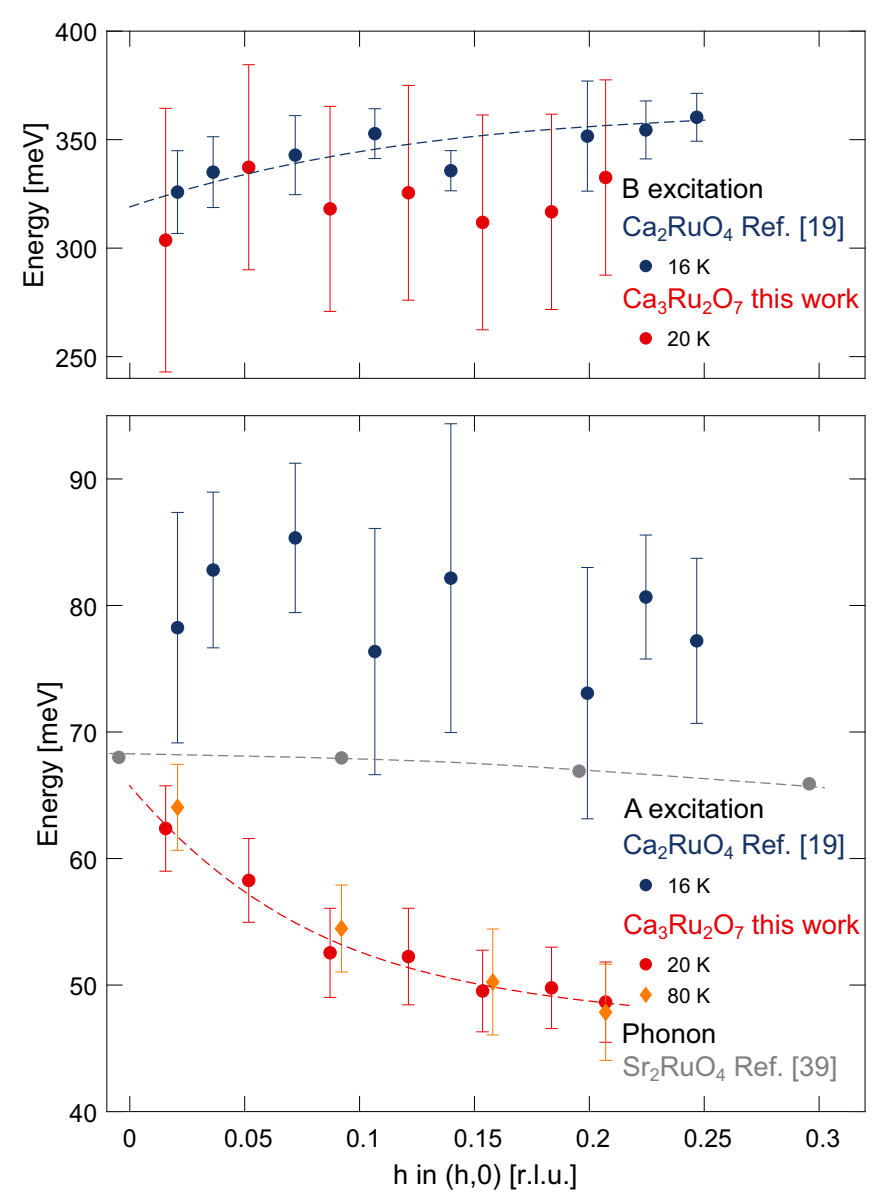

FIG. 4. Dispersion of the low-energy excitations along $\theta$ which corresponds to a varying in-plane momentum $\mathbf{q}=(h, 0)$ for the $\mathrm{A}$ (bottom) and B (top) excitations. Error bars for the A excitation in $\mathrm{Ca}_{3} \mathrm{Ru}_{2} \mathrm{O}_{7}$ indicate standard deviation $3 \sigma$ of the fitted peak position. By contrast, for the B excitation, the error bars reflect the peak width at $98 \%$ of the total height. Dark blue data show the dispersion for the single layer $\mathrm{Ca}_{2} \mathrm{RuO}_{4}$, taken from Ref. [19] including error bars. Gray data show a phonon dispersion in $\mathrm{Sr}_{2} \mathrm{RuO}_{4}$ measured by inelastic neutron scattering [39]. Dashed lines are guides to the eye.

$\mathrm{Ca}_{2} \mathrm{RuO}_{4}$ no dispersion was resolved for the corresponding branch.

To gain insight into the microscopic picture behind these excitations, the RIXS response was modeled for both compounds, and compared to the experimental spectra in Fig. 2. We used the fast collision approximation $[25,26]$ of the RIXS cross section describing the light-induced excitation-and subsequent absorption - of an electron from the $\mathrm{O} 1 s$ level into the $2 p$ level, for both LV and LH incoming polarization. Full detailed description of this approach is reported in Appendixes A and B. The RIXS intensity was calculated via exact diagonalization of a model Hamiltonian defined on a cluster of two ruthenium sites connected by one planar oxygen site along an in-plane direction. The bond bending due to the rotation of the octahedra around the $c$ axis is allowed. The ruthenium-site Hamiltonian is defined on the $t_{2 g}$ subspace and consists of three terms: (1) CF splitting $\Delta$ between the $d_{x y}$ and $d_{x z}, d_{y z}$ orbitals, (2) SOC $\lambda$, and (3) Coulomb interaction, which is expanded into intraorbital and interorbital Hubbard interactions of strengths $U$ and $\left(U-5 J_{\mathrm{H}} / 2\right)$, respectively. Interorbital Hund's coupling as well as the pair-hopping term are both of strength $J_{\mathrm{H}}$. Material specific values $\Delta=0.3 \mathrm{eV}$, $\lambda=0.05 \mathrm{eV}, U=2.0 \mathrm{eV}$, and $J_{\mathrm{H}}=0.4 \mathrm{eV}$ [20,41-43] are used to evaluate the model for both $\mathrm{Ca}_{2} \mathrm{RuO}_{4}$ and $\mathrm{Ca}_{3} \mathrm{Ru}_{2} \mathrm{O}_{7}$. Similar values of $\Delta, U$, and $J_{\mathrm{H}}$ have been used for DMFT calculations [44] of $\mathrm{Ca}_{2} \mathrm{RuO}_{4}$ and are comparable to those used in modeling the spin-excitation dispersion observed by neutron scattering [17] and RIXS spectra [19], as well as magnetic anisotropy [45]. Here, we point out that small differences from previous estimates of the microscopic parameters are fully awaited since, in our description, the oxygen degrees of freedom are explicitly included, and this may lead to a renormalization of the local interaction terms. To take into account the different ground states, $\mathrm{Ca}_{2} \mathrm{RuO}_{4}$ is modeled with an antiferromagnetic (AFM) in-plane interaction, whereas we consider an extra exchange field to stabilize the ferromagnetic (FM) ground state and spins along the in-plane easy axis in $\mathrm{Ca}_{3} \mathrm{Ru}_{2} \mathrm{O}_{7}[12,16]$. Henceforth, we will also refer to the $\mathrm{Ca}_{2} \mathrm{RuO}_{4}$ and $\mathrm{Ca}_{3} \mathrm{Ru}_{2} \mathrm{O}_{7}$ bonds as $\mathrm{AFM}$ and $\mathrm{FM}$, respectively, corresponding to the G-type and A-type AFM structures.

In Fig. 2(c) the calculated RIXS responses in LH and LV polarizations are presented for both ground states, showing a reasonable overall agreement with the experimental spectra in Fig. 2(b). In both cases four distinct excitations are evident, with approximate energy losses of $0.08,0.4,1-1.5$, and $2 \mathrm{eV}$. In the FM case, we observe an overall decrease of the peak intensities. This effect is even more pronounced for excitations above $1 \mathrm{eV}$.

The origin of the four features for $\mathrm{Ca}_{2} \mathrm{RuO}_{4}$ has already been assigned in a previous work, and we recall it here for convenience [19]. We point out that, in the present simulation, the RIXS intensity has been evaluated by fully taking into account the scattering geometry in Fig. 1(a), and that the LV and LH spectra have been obtained by averaging the spectra over two orthogonal in-plane bond directions of the cluster.

Excitations $\mathrm{A}, \mathrm{B}, \mathrm{C}$, and $\mathrm{D}$ in $\mathrm{Ca}_{2} \mathrm{RuO}_{4}$ have been interpreted on the basis of the multiplet structure of the $d^{4}$ configuration of the $\mathrm{Ru}^{4+}$ ion. In particular, they are associated to transitions within low-energy spin-orbital configurations, which have one doubly occupied orbital (doublon) or two doubly occupied orbitals. In the framework of the ionic picture, structures $\mathrm{C}$ and $\mathrm{D}$ have been assigned to $J_{H}$ driven spin-state transitions between $S=1$ and $S=0$ states in the single- and two-doublon sectors [19]. The partial suppression of the weight associated to the features above $1 \mathrm{eV}$ in the case of a FM configuration is a consequence of the Pauli blocking of those intra- $t_{2 g}$ transitions. This mechanism may justify the lowered intensity of $\mathrm{C}$ and $\mathrm{D}$ structures in the FM background of $\mathrm{Ru}-\mathrm{O}$ planes in $\mathrm{Ca}_{3} \mathrm{Ru}_{2} \mathrm{O}_{7}$. We notice that the experimental results suggest a stronger suppression than predicted by the model calculations. A reason for the discrepancies between experiment and the modeling may have to do with the less insulating ground state of $\mathrm{Ca}_{3} \mathrm{Ru}_{2} \mathrm{O}_{7}$. The cluster calculations may not capture precisely the more delocalized nature of $\mathrm{Ca}_{3} \mathrm{Ru}_{2} \mathrm{O}_{7}$.

In $\mathrm{Ca}_{2} \mathrm{RuO}_{4}$, the lowest energy features $\mathrm{A}$ and $\mathrm{B}$ are associated to spin-orbital excitations within the $S=1$ subspace 
of the $t_{2 g}$ multiplets, whose energies are determined by the relative strength of the CF potential and the SOC. Even though they are not fully resolved experimentally due to limited energy resolution, those excitations are accessible in oxygen $K$ edge because of the SOC in the Ru $4 d$ shell which strongly hybridizes $4 d$ states with $\mathrm{O} 2 p$ orbitals. In particular, the B structure has been attributed to multiple transitions to the highest energy $S=1$ spin-orbital sector, while the A structure has been generically associated to composite magnetic transitions within the lowest-energy sector. We observe that, in the FM case, features occurring at a similar energy scale are observed, and we want to elucidate the possible spin-orbital (magnetic) origin of these excitations, with a special focus on the lowest A feature.

Beforehand, we observe that the lowest lying excitation A has an energy scale typical of both optical phonons and magnons. The strong dispersion of this excitation near the zone center is, however, atypical for optical phonons. In $\mathrm{Sr}_{2} \mathrm{RuO}_{4}$, where complete phonon dispersions have been calculated and probed by neutron scattering [39,46], optical phonons are in fact found at $\sim 70$ and $\sim 90 \mathrm{meV}$. None of them has a dispersion around the zone center compatible with what we observe in $\mathrm{Ca}_{3} \mathrm{Ru}_{2} \mathrm{O}_{7}$. This is demonstrated in Fig. 4, where the relevant optical $\mathrm{Sr}_{2} \mathrm{RuO}_{4}$ phonon dispersionmeasured by neutron scattering-is shown in gray. In comparison to the $\mathrm{A}$ excitation measured in $\mathrm{Ca}_{3} \mathrm{Ru}_{2} \mathrm{O}_{7}$, this optical phonon is nondispersive. Moreover, the $70 \mathrm{meV}$ optical phonon found in $\mathrm{Sr}_{2} \mathrm{RuO}_{4}$ stems from vibrations of the apical oxygen, whereas we are probing on the planar and interplanar oxygen sites. On this basis, assigning a magnetic origin to the lowest lying excitation appears the most plausible interpretation. We stress that it is not unusual to observe magnetic excitations beyond the magnetic ordered state due to the persistence of short-range magnetic correlations. In cuprates and iron pnictides, paramagnon excitations are found deep into the magnetically disordered state [47-49]. Observing no significant temperature dependence of the A excitation dispersion is therefore expected.

To further verify the magnetic origin of the A excitations in $\mathrm{Ca}_{2} \mathrm{RuO}_{4}$ and $\mathrm{Ca}_{3} \mathrm{Ru}_{2} \mathrm{O}_{7}$, and to reveal their distinct nature, we evaluated the dynamic spin structure factors $S^{\mu}(\mathbf{q}, \omega)$, and spin-spin dynamic spin structure factors $\left(S_{i} \cdot S_{i+1}\right)^{\mu}(\mathbf{q}, \omega)$, $\mu=x, y, z$ [Figs. 5(b), 5(c) 5(e), and 5(f)] for $\mathbf{q}=(0,0)$ and $(\pi, 0)$, which are the only viable values for the momentum transfer of our Ru-O-Ru cluster. Here, we point out that the ground state is made by magnetic moments that are aligned in the $\mathrm{Ru}$ plane. For convenience, we refer to $\|, \perp, z$ for a spin mode excitation that is collinear, perpendicular in plane, and perpendicular out of plane with respect to the orientation of the ordered magnetic moments in the ground state, respectively.

Let us start with the AFM case in Figs. 5(a)-5(c). The comparison of the low-energy part of the RIXS spectrum for the AFM configuration with the calculated spin structure factors at $\mathbf{q}=(0,0)$ allows one to associate the dominant excitation in the RIXS spectrum to features with transverse single spin modes, i.e., $S^{z}$ and $S^{\perp}$, and longitudinal twospin $S^{\|} S^{\|}$correlation functions. This is consistent with the previous interpretation of the A excitation as evidence of composite excitations such as longitudinal (Higgs) two-particle
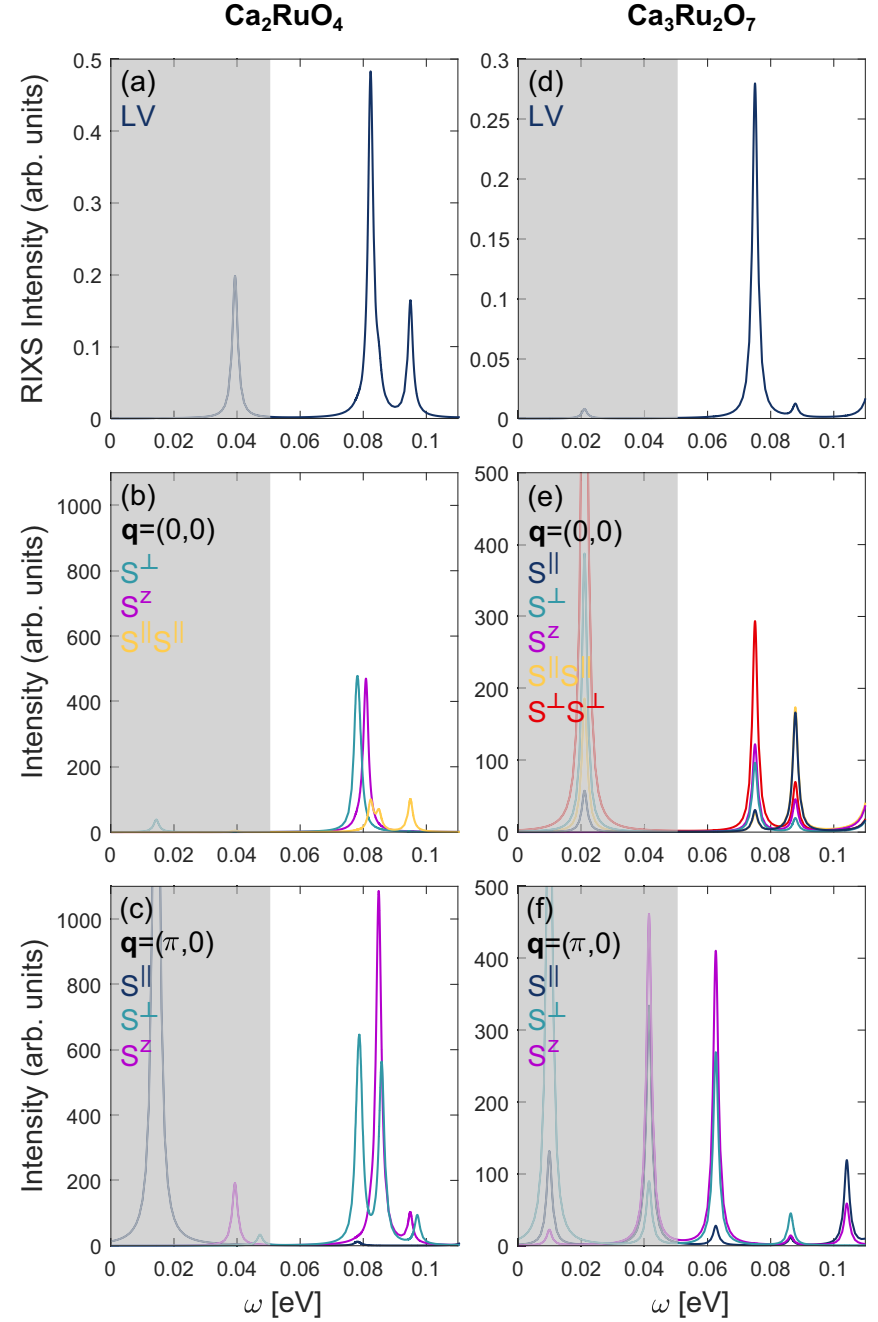

FIG. 5. Theoretical model calculation of the RIXS spectra in panels (a) and (d) and the dynamic spin structure factors $S^{\mu}(\mathbf{q}, \omega)$, and spin-spin dynamic spin structure factors $\left(S_{i} \cdot S_{i+1}\right)^{\mu}(\mathbf{q}, \omega)$, $\mu=\|, \perp, z$, for $\mathbf{q}=(0,0)$ and $(\pi, 0)$ in the low energy regime for $\mathrm{Ca}_{2} \mathrm{RuO}_{4}$ and $\mathrm{Ca}_{3} \mathrm{Ru}_{2} \mathrm{O}_{7}$. The gray shaded area corresponds to the energy region currently not accessible by RIXS due to the limited resolution.

and transverse bimagnon modes [18,19]. In particular, from the analysis we observe that the lowest energy spin excitations occur at about 20 and $40 \mathrm{meV}$-see Figs. 5(b) and 5(c), mainly through single spin flip at each Ru site. The former energy scale is related to the effective single ion anisotropy due to the interplay of spin-orbit and crystal field potential. A distinctive aspect of the magnetic ground state is that, due to the spin-orbit coupling and crystal field potential, there is neither rotational nor parity conservation for the local spin. The resulting ground state is then a quantum superposition of several components. Specifically, it consists of dominant exchange driven anisotropic antiferromagnetic correlations, and it also includes states corresponding to the variation of amplitude and direction of the local $S=1$ magnetic moments with respect to the easy axis. This peculiar character of the ground state allows one to have a significant spectral weight associated to high-energy excitations, corresponding to the 
RIXS active states close to $80 \mathrm{meV}$-see Fig. 5(a). Taking into account the energy profile of the dynamic spin response, we deduce that the modes at about $80 \mathrm{meV}$ have a multiparticle spin character, as they are accessible by means of both single transverse and double longitudinal spin excitations. Our results also predict the existence of a lower energy feature in the RIXS spectrum, having similar character, at $40 \mathrm{meV}$, in an energy range which is not detectable in the present experiment.

The FM case offers a similar result, since the lowest A feature may also be associated to magnetic excitations. Notably, in this case, the dominant excitation occurs at slightly lower energy, and corresponds to mainly transverse spin excitations, of single- $S^{z}$ and $S^{\perp}$ or two-particle type $S^{\perp} S^{\perp}$. Moreover, according to the simulation, the existence of a very weak feature located at $20 \mathrm{meV}$ is also predicted.

Having identified the nature of the magnetic excitations associated to the lowest RIXS feature in both the AFM and FM ground states, one can also estimate the bandwidth of the continuum of the corresponding collective modes propagating along the $(0,0) \rightarrow(\pi, 0)$ path. Comparing the relevant $S^{\mu}(\mathbf{q}, \omega)$ at $\mathbf{q}=(\pi, 0)$ and $(0,0)$ shows that, in the AFM case, magnetic peaks are located approximately in the same energy range at different wave vectors. On the contrary, in the FM configuration, the peaks associated to the single spin excitations are shifted to lower energies by $20-30 \mathrm{meV}$, when going from $\mathbf{q}=(0,0)$ to $\mathbf{q}=(\pi, 0)$. This is in accordance with what is observed in the experimental spectra-see Fig. 4. We also carried out the calculation of the local and two-site orbital angular momentum correlation functions, which reveal that the A peak in the AFM case has significant orbital contribution, while it is substantially suppressed in the FM case. This is consistent with the observation that the FM ground state has a different orbital pattern [50] when compared to the AFM configuration. The doublon can have a stronger tendency to occupy different orbitals on neighboring Ru sites in the FM case. Moreover, the spin-orbit coupling tends to align the orbital moments, since the Ru spins are also ferromagnetically correlated. This implies that orbital variations can be suppressed in the low-energy spin sector. Here, we argue that the lack of the orbital component in the targeted excitation allows one to have a larger effective exchange, which results in an enhancement of the bandwidth as we find in the cluster analysis.

\section{CONCLUSIONS}

In summary, we have carried out a combined oxygen $K$ edge XAS and RIXS study of $\mathrm{Ca}_{3} \mathrm{Ru}_{2} \mathrm{O}_{7}$. Our results are compared to (i) equivalent experimental results previously obtained on single layer $\mathrm{Ca}_{2} \mathrm{RuO}_{4}$ and (ii) local cluster modeling of $\mathrm{Ca}_{3} \mathrm{Ru}_{2} \mathrm{O}_{7}$ and $\mathrm{Ca}_{2} \mathrm{RuO}_{4}$. In particular, the oxygen $K$-edge RIXS spectra are fundamentally different in $\mathrm{Ca}_{3} \mathrm{Ru}_{2} \mathrm{O}_{7}$ and $\mathrm{Ca}_{2} \mathrm{RuO}_{4}$, reflecting their different ground states. Whereas in $\mathrm{Ca}_{2} \mathrm{RuO}_{4}$ a set of excitations within the $t_{2 g}$ subspace, consisting of two low-energy and two mid/high-energy structures, is observed, only the two lowest intra- $t_{2 g}$ excitations have a significant amplitude in $\mathrm{Ca}_{3} \mathrm{Ru}_{2} \mathrm{O}_{7}$. This effect is captured by the local cluster modeling taking into account the different in-plane magnetic couplings. Finally, we demonstrated that the lowest lying intra- $t_{2 g}$ excitation in $\mathrm{Ca}_{3} \mathrm{Ru}_{2} \mathrm{O}_{7}$ is dispersing, revealing its collective origin. We argue, based on the exact dispersion and comparison to spin correlation function computations, that this excitation is magnonic rather than phononic in nature. In fact, it is suggested to be dominantly a transverse mode with multiparticle character, which is indirectly allowed at the oxygen $K$ edge through substantial SOC of Ru ions.

\section{ACKNOWLEDGMENTS}

The experimental work was performed at the ADRESS beamline of the SLS at the Paul Scherrer Institut, Villigen PSI, Switzerland. We thank the ADRESS beamline staff for technical support. K.v.A., M.H., Q.W, L.D., O.I., and J.C. acknowledge support by the Swiss National Science Foundation. M.C. and F.F. acknowledge support by the project "Two-dimensional Oxides Platform for SPIN-orbitronics nanotechnology (TOPSPIN)" funded by the MIUR (PRIN) Bando 2017, Grant No. 20177SL7HC. Y.S. is funded by the Swedish Research Council (VR) with a Starting Grant (No. 201705078). Work at the Paul Scherrer Institut has been funded by the Swiss National Science Foundation through the Sinergia network Mott Physics Beyond the Heisenberg (MPBH) model (SNSF Research Grants No. CRSII2_141962 and No. CRSII2_160765).

\section{APPENDIX A: MODEL HAMILTONIAN}

We report here the details of the microscopic model describing the energy levels and wave functions of the considered Ru-O-Ru cluster. The examined Hamiltonian [51,52] is expressed as

$$
H=H_{\mathrm{kin}}+H_{e l-e l}+H_{c f}+H_{\mathrm{soc}}+H_{m} .
$$

The first term in Eq. (A1) is the kinetic operator describing the Ru-O connectivity:

$$
H_{\mathrm{kin}}=\sum_{i j, \alpha \beta, \sigma} t_{i j}^{\alpha \beta}\left(p_{i \alpha \sigma}^{\dagger} d_{j \beta \sigma}+\text { H.c. }\right)
$$

where $d_{i \beta \sigma}^{\dagger}$ is the creation operator for an electron with spin $\sigma$ at the $i$ site in the $\beta$ orbital of the $t_{2 g}$ sector $\left(d_{x y}, d_{x z}, d_{y z}\right)$, while $p_{i \alpha \sigma}$ is the annihilation operator of an electron with spin $\sigma$ at the $i$ site in the $\alpha$ orbital of the $\left(p_{x}, p_{y}, p_{z}\right)$ space of the oxygen. Hopping amplitudes $t_{i j}^{\alpha \beta}$ include all the allowed symmetry terms according to the Slater-Koster rules $[53,54]$ for a given bond connecting a ruthenium to an oxygen atom along, say, the $x$ direction. We allow for the relative rotation of the oxygen octahedra surrounding the Ru site, assuming that the Ru-O-Ru bond can form an angle $\theta=\left(180^{\circ}-\phi\right)$. The case with $\phi=0^{\circ}$ corresponds to the tetragonal undistorted bond, while a nonvanishing value of $\phi$ arises when the $\mathrm{RuO}_{6}$ octahedra are rotated of the corresponding angle around the $c$ axis.

The second term is the Coulomb interaction, which is expressed in terms of Kanamori parameters $U, U^{\prime}$, and $J_{H}$ as 
follows:

$$
\begin{aligned}
H_{e l-e l}= & U \sum_{i \alpha} n_{i \alpha \uparrow} n_{i \alpha \downarrow}-2 J_{H} \sum_{i \alpha \beta} \mathbf{S}_{i \alpha} \cdot \mathbf{S}_{i \beta} \\
& +\left(U^{\prime}-\frac{J_{H}}{2}\right) \sum_{i \alpha \neq \beta} n_{i \alpha} n_{i \beta} \\
& +J^{\prime} \sum_{i \alpha \beta} d_{i \alpha \uparrow}^{\dagger} d_{i \alpha \downarrow}^{\dagger} d_{i \beta \uparrow} d_{i \beta \downarrow},
\end{aligned}
$$

where $n_{i \alpha \sigma}, \mathbf{S}_{i \alpha}$ are the on site charge for spin $\sigma$ and the spin operators for the $\alpha$ orbital, respectively. $U\left(U^{\prime}\right)$ is the intra (inter) -orbital Coulomb repulsion, $J_{H}$ is the Hund coupling, and $J^{\prime}$ the pair hopping term. Due to the invariance for rotations in the orbital space, the following relations hold: $U=U^{\prime}+2 J_{H}$; $J^{\prime}=J_{H}$.

The $H_{c f}$ part of the Hamiltonian $H$ is the crystalline field potential, controlling the symmetry lowering from the cubic to tetragonal one, due to the compression of $\mathrm{RuO}_{6}$ octahedra along the $c$ axis:

$$
H_{c f}=\sum_{i} \Delta_{i}\left[n_{i x y}-\frac{1}{2}\left(n_{i x z}+n_{i y z}\right)\right]
$$

The SOC Hamiltonian reads as

$$
H_{\mathrm{soc}}=\lambda \sum_{i} \mathbf{L}_{i} \cdot \mathbf{S}_{i}
$$

Due to the cubic $\mathrm{CF}$ terms in $\mathrm{RuO}_{6}$ octahedra separating the lower $t_{2 g}$ from the unoccupied $e_{g}$ levels, $\mathbf{L}_{\mathbf{i}}$ stands for the angular momentum operator projected onto the $t_{2 g}$ subspace. Its components have the following expression in terms of orbital fermionic operators:

$$
\begin{aligned}
L_{i x} & =i \sum_{\sigma}\left[d_{i x y \sigma}^{\dagger} d_{i x z \sigma}-d_{i x z \sigma}^{\dagger} d_{i x y \sigma}\right], \\
L_{i y} & =i \sum_{\sigma}\left[d_{i y z \sigma}^{\dagger} d_{i x y \sigma}-d_{i x y \sigma}^{\dagger} d_{i y z \sigma}\right], \\
L_{i z} & =i \sum_{\sigma}\left[d_{i x z \sigma}^{\dagger} d_{i y z \sigma}-d_{i y z \sigma}^{\dagger} d_{i x z \sigma}\right] .
\end{aligned}
$$

Finally, $H_{m}$ in Eq. (A1) is an effective exchange field which pins the magnetization at the Ru sites to be in the $(x, y)$ plane for the FM ground state:

$$
H_{m}=\sum_{i} \mathbf{S}_{i} \cdot \mathbf{B}_{x y}
$$

\section{APPENDIX B: CALCULATION OF THE RIXS CROSS SECTION}

The RIXS intensity is described by the KramersHeisenberg relation

$I\left(\omega, \mathbf{q}, \epsilon, \epsilon^{\prime}\right)=\sum_{f}\left|A_{f g}\left(\omega, \mathbf{q}, \epsilon, \epsilon^{\prime}\right)\right|^{2} \delta\left(E_{f}+\omega_{k^{\prime}}-E_{g}-\omega_{k}\right)$,

where $\omega=\omega_{k^{\prime}}-\omega_{k}$ and $\mathbf{q}=\mathbf{k}^{\prime}-\mathbf{k}$ stand for the energy and momentum transferred by the scattered photon and $\epsilon$ and $\epsilon^{\prime}$ for the incoming and outgoing light polarization vectors. We

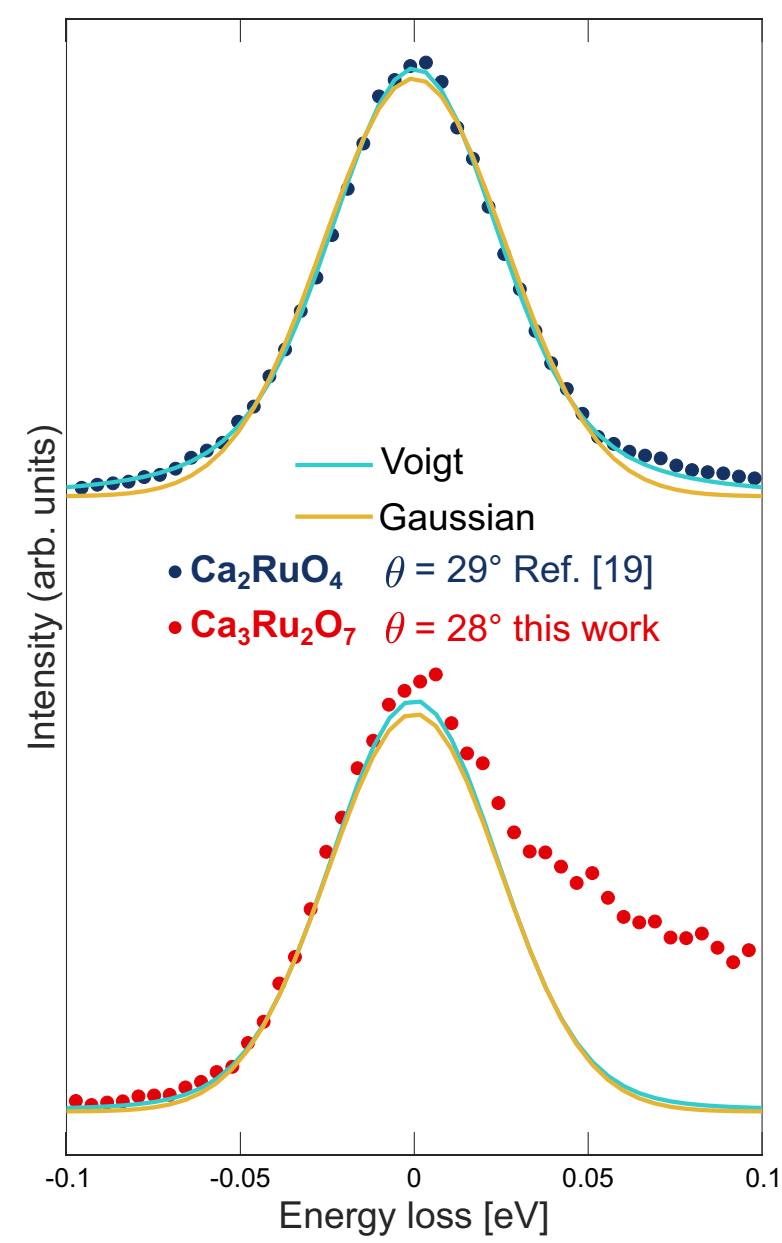

FIG. 6. Analysis of elastic scattering. Elastic scattering part of the RIXS spectra recorded on $\mathrm{Ca}_{2} \mathrm{RuO}_{4}$ (top curve, taken from Ref. [19]) and $\mathrm{Ca}_{3} \mathrm{Ru}_{2} \mathrm{O}_{7}$ (bottom curve) near specular condition as indicated. Solid lines are Gaussian and Voigt fits. In both profiles, the Gaussian width is fixed to the energy resolution of the experiment. On $\mathrm{Ca}_{3} \mathrm{Ru}_{2} \mathrm{O}_{7}$, the full widths at half maximum (FWHM) of the Gaussian and Lorentzian contributions in the Voigt profile are $53.3 \mathrm{meV}$ and $6.9 \mathrm{meV}$, respectively. To enhance visibility, the spectra are normalized to their maximum and the spectra and fits of $\mathrm{Ca}_{2} \mathrm{RuO}_{4}$ are given a vertical shift.

adopt the dipole and fast collision approximation, in which the RIXS scattering amplitude is reduced to

$$
A_{f g}=\frac{1}{i \Gamma}\left\langle f\left|R\left(\epsilon, \epsilon^{\prime}, \mathbf{q}\right)\right| g\right\rangle,
$$

where $R$ is the effective RIXS scattering operator describing two subsequent dipole transitions and $\Gamma$ is the core-hole broadening. In the oxygen $K$-edge RIXS, the dipole transitions create an $\mathrm{O} 1 s$ core hole and extra valence electron in a $2 p$ orbital and vice versa, and the scattering operator has the following expression:

$$
R\left(\epsilon_{\nu}, \epsilon_{v^{\prime}}\right) \propto \sum_{i, \sigma} e^{i \mathbf{q} \cdot \mathbf{r}_{i}} p_{\nu^{\prime} \sigma} p_{\nu \sigma}
$$

where $v$ is the $(x, y, z)$ orbital and the sum over the different spin states is assumed. Matrix elements are then evaluated among oxygen valence states in Eq. (B2). Notably, the valence 
electron in a $2 p$ orbital hybridizes and interacts with the Ru $d$ electrons.

In the adopted experimental scattering geometry, the dependence upon the incident angle $\theta_{\text {in }}$ and scattering angle $\alpha=$ $130^{\circ}$ between the incoming/outgoing polariization vectors is

$$
\begin{aligned}
\epsilon_{L H} & =\epsilon_{x} \cos \theta_{\text {in }}+\epsilon_{z} \sin \theta_{\text {in }}, \\
\epsilon_{L V} & =\epsilon_{y}, \\
\epsilon^{\prime} & =\epsilon_{x}^{\prime} \cos \left(\theta_{\text {in }}+\alpha\right)+\epsilon_{y}^{\prime}+\epsilon_{z}^{\prime} \sin \left(\theta_{\text {in }}+\alpha\right) .
\end{aligned}
$$

Here the coordinate frame $(x, y, z)$ corresponds to the tetragonal axis frame $\left(a_{T}, b_{T}, c\right)$. Since the outgoing polarization is not resolved, the RIXS intensity is obtained by summing up incoherently over all three polarization directions $\left(\epsilon_{x}^{\prime}, \epsilon_{y}^{\prime}, \epsilon_{z}^{\prime}\right)$.

\section{APPENDIX C: SUBTRACTION OF ELASTIC SCATTERING CONTRIBUTIONS}

The analysis of the lowest lying (dispersive) A excitation in $\mathrm{Ca}_{3} \mathrm{Ru}_{2} \mathrm{O}_{7}$ involves modeling of the elastic scattering. By assuming that the intrinsic elastic scattering line shape is Lorentzian and that the finite energy resolution is accounted for by a Gaussian convolution, the effective line shape would therefore theoretically be a Voigt function. In practice, the Gaussian component is dominating over the Lorentzian. Therefore, both Gaussian and Voigt functions can be used to model elastic scattering.

In Fig. 6, elastic scattering profiles measured on $\mathrm{Ca}_{2} \mathrm{RuO}_{4}$ and $\mathrm{Ca}_{3} \mathrm{Ru}_{2} \mathrm{O}_{7}$ are shown and compared to fits using Gaussian and Voigt profiles. The counting statistic is better for the data recorded on $\mathrm{Ca}_{2} \mathrm{RuO}_{4}$. There, the fitting indeed suggests that the Voigt profile provides a slightly better description of the elastic line. This is to be expected as the fitting to a Voigt function involves an additional fitting parameter. For $\mathrm{Ca}_{3} \mathrm{Ru}_{2} \mathrm{O}_{7}$, the analysis is harder since (a) the counting statistics are less good, (b) the lowest lying excitation overlaps partially with the elastic scattering, and (c) the elastic contribution in the spectra is overall much smaller than in $\mathrm{Ca}_{2} \mathrm{RuO}_{4}$. As shown in Fig. 6, both Voigt and Gaussian profiles provide good description of the elastic scattering. The difference between these two profiles is marginal and negligible compared to the intensity of the lowest lying excitation. To be consistent over all incident angles, the analysis of $\mathrm{Ca}_{3} \mathrm{Ru}_{2} \mathrm{O}_{7}$ data presented in this paper made use of a Gaussian profile. Data presented in Ref. [19] on $\mathrm{Ca}_{2} \mathrm{RuO}_{4}$ is fitted to a Voigt function. The choice of Gaussian or Voigt function for the modeling of elastic scattering has no impact on the derived conclusions.
[1] J. F. Karpus, R. Gupta, H. Barath, S. L. Cooper, and G. Cao, Phys. Rev. Lett. 93, 167205 (2004).

[2] X. N. Lin, Z. X. Zhou, V. Durairaj, P. Schlottmann, and G. Cao, Phys. Rev. Lett. 95, 017203 (2005).

[3] W. Bao, Z. Q. Mao, Z. Qu, and J. W. Lynn, Phys. Rev. Lett. 100, 247203 (2008).

[4] N. Kikugawa, A. Winfried Rost, C. William Hicks, A. John Schofield, and A. Peter Mackenzie, J. Phys. Soc. Jpn. 79, 024704 (2010).

[5] M. Zhu, J. Peng, T. Zou, K. Prokes, S. D. Mahanti, T. Hong, Z. Q. Mao, G. Q. Liu, and X. Ke, Phys. Rev. Lett. 116, 216401 (2016).

[6] H. Xing, L. Wen, C. Shen, J. He, X. Cai, J. Peng, S. Wang, M. Tian, Z.-A. Xu, W. Ku, Z. Mao, and Y. Liu, Phys. Rev. B 97, 041113(R) (2018).

[7] C. Sow, R. Numasaki, G. Mattoni, S. Yonezawa, N. Kikugawa, S. Uji, and Y. Maeno, Phys. Rev. Lett. 122, 196602 (2019).

[8] D. Puggioni, M. Horio, J. Chang, and J. M. Rondinelli, Phys. Rev. Research 2, 023141 (2020).

[9] G. Cao, L. Balicas, Y. Xin, J. E. Crow, and C. S. Nelson, Phys. Rev. B 67, 184405 (2003).

[10] Y. Yoshida, I. Nagai, S.-I. Ikeda, N. Shirakawa, M. Kosaka, and N. Môri, Phys. Rev. B 69, 220411(R) (2004).

[11] E. Ohmichi, Y. Yoshida, S. I. Ikeda, N. Shirakawa, and T. Osada, Phys. Rev. B 70, 104414 (2004).

[12] Y. Yoshida, S.-I. Ikeda, H. Matsuhata, N. Shirakawa, C. H. Lee, and S. Katano, Phys. Rev. B 72, 054412 (2005).

[13] S. Nakatsuji, V. Dobrosavljević, D. Tanasković, M. Minakata, H. Fukazawa, and Y. Maeno, Phys. Rev. Lett. 93, 146401 (2004).

[14] K. Takenaka, Y. Okamoto, T. Shinoda, N. Katayama, and Y. Sakai, Nat. Commun. 8, 14102 (2017).
[15] O. Friedt, M. Braden, G. André, P. Adelmann, S. Nakatsuji, and Y. Maeno, Phys. Rev. B 63, 174432 (2001).

[16] M. Braden, G. André, S. Nakatsuji, and Y. Maeno, Phys. Rev. B 58, 847 (1998).

[17] A. Jain, M. Krautloher, J. Porras, G. H. Ryu, D. P. Chen, D. L. Abernathy, J. T. Park, A. Ivanov, J. Chaloupka, G. Khaliullin, B. Keimer, and B. J. Kim, Nat. Phys. 13, 633 (2017).

[18] S.-M. Souliou, J. Chaloupka, G. Khaliullin, G. Ryu, A. Jain, B. J. Kim, M. Le Tacon, and B. Keimer, Phys. Rev. Lett. 119, 067201 (2017).

[19] L. Das, F. Forte, R. Fittipaldi, C. G. Fatuzzo, V. Granata, O. Ivashko, M. Horio, F. Schindler, M. Dantz, Y. Tseng, D. E. McNally, H. M. Rønnow, W. Wan, N. B. Christensen, J. Pelliciari, P. Olalde-Velasco, N. Kikugawa, T. Neupert, A. Vecchione, T. Schmitt, M. Cuoco, and J. Chang, Phys. Rev. X 8, 011048 (2018).

[20] C. G. Fatuzzo, M. Dantz, S. Fatale, P. Olalde-Velasco, N. E. Shaik, B. Dalla Piazza, S. Toth, J. Pelliciari, R. Fittipaldi, A. Vecchione, N. Kikugawa, J. S. Brooks, H. M. Rønnow, M. Grioni, C. Rüegg, T. Schmitt, and J. Chang, Phys. Rev. B 91, 155104 (2015).

[21] V. Bisogni, L. Simonelli, L. J. P. Ament, F. Forte, M. Moretti Sala, M. Minola, S. Huotari, J. van den Brink, G. Ghiringhelli, N. B. Brookes, and L. Braicovich, Phys. Rev. B 85, 214527 (2012).

[22] M. Moretti Sala, M. Rossi, S. Boseggia, J. Akimitsu, N. B. Brookes, M. Isobe, M. Minola, H. Okabe, H. M. Rønnow, L. Simonelli, D. F. McMorrow, and G. Monaco, Phys. Rev. B 89, 121101(R) (2014).

[23] X. Lu, P. Olalde-Velasco, Y. Huang, V. Bisogni, J. Pelliciari, S. Fatale, M. Dantz, J. G. Vale, E. C. Hunter, J. Chang, V. N. 
Strocov, R. S. Perry, M. Grioni, D. F. McMorrow, H. M. Rønnow, and T. Schmitt, Phys. Rev. B 97, 041102(R) (2018).

[24] D. Pincini, L. S. I. Veiga, C. D. Dashwood, F. Forte, M. Cuoco, R. S. Perry, P. Bencok, A. T. Boothroyd, and D. F. McMorrow, Phys. Rev. B 99, 075125 (2019).

[25] L. J. P. Ament, F. Forte, and J. van den Brink, Phys. Rev. B 75, 115118 (2007).

[26] L. J. P. Ament, M. van Veenendaal, T. P. Devereaux, J. P. Hill, and J. van den Brink, Rev. Mod. Phys. 83, 705 (2011).

[27] H. Fukazawa, S. Nakatsuji, and Y. Maeno, Physica B 281, 613 (2000).

[28] S. Nakatsuji and Y. Maeno, J. Solid State Chem. 156, 26 (2001).

[29] G. Ghiringhelli, A. Piazzalunga, C. Dallera, G. Trezzi, L. Braicovich, T. Schmitt, V. N. Strocov, R. Betemps, L. Patthey, X. Wang, and M. Grioni, Rev. Sci. Instrum. 77, 113108 (2006).

[30] V. Strocov, T. Schmitt, U. Flechsig, T. Schmidt, A. Imhof, Q. Chen, J. Raabe, R. Betemps, D. Zimoch, J. Krempasky et al., J. Synchrotron Radiat. 17, 631 (2010).

[31] C. T. Chen, L. H. Tjeng, J. Kwo, H. L. Kao, P. Rudolf, F. Sette, and R. M. Fleming, Phys. Rev. Lett. 68, 2543 (1992).

[32] H.-J. Noh, S.-J. Oh, B.-G. Park, J.-H. Park, J.-Y. Kim, H.-D. Kim, T. Mizokawa, L. H. Tjeng, H.-J. Lin, C. T. Chen, S. Schuppler, S. Nakatsuji, H. Fukazawa, and Y. Maeno, Phys. Rev. B 72, 052411 (2005).

[33] M. Malvestuto, E. Carleschi, R. Fittipaldi, E. Gorelov, E. Pavarini, M. Cuoco, Y. Maeno, F. Parmigiani, and A. Vecchione, Phys. Rev. B 83, 165121 (2011).

[34] M. Malvestuto, V. Capogrosso, E. Carleschi, L. Galli, E. Gorelov, E. Pavarini, R. Fittipaldi, F. Forte, M. Cuoco, A. Vecchione, and F. Parmigiani, Phys. Rev. B 88, 195143 (2013).

[35] E. B. Guedes, M. Abbate, K. Ishigami, A. Fujimori, K. Yoshimatsu, H. Kumigashira, M. Oshima, F. C. Vicentin, P. T. Fonseca, and R. J. O. Mossanek, Phys. Rev. B 86, 235127 (2012).

[36] A. Koga, N. Kawakami, T. M. Rice, and M. Sigrist, Phys. Rev. Lett. 92, 216402 (2004).

[37] A. Liebsch and H. Ishida, Phys. Rev. Lett. 98, 216403 (2007).

[38] E. Gorelov, M. Karolak, T. O. Wehling, F. Lechermann, A. I. Lichtenstein, and E. Pavarini, Phys. Rev. Lett. 104, 226401 (2010).

[39] M. Braden, W. Reichardt, Y. Sidis, Z. Mao, and Y. Maeno, Phys. Rev. B 76, 014505 (2007).
[40] H. Gretarsson, H. Suzuki, H. Kim, K. Ueda, M. Krautloher, B. J. Kim, H. Yavas, G. Khaliullin, and B. Keimer, Phys. Rev. B 100, 045123 (2019).

[41] T. Mizokawa, L. H. Tjeng, G. A. Sawatzky, G. Ghiringhelli, O. Tjernberg, N. B. Brookes, H. Fukazawa, S. Nakatsuji, and Y. Maeno, Phys. Rev. Lett. 87, 077202 (2001).

[42] C. N. Veenstra, Z.-H. Zhu, M. Raichle, B. M. Ludbrook, A. Nicolaou, B. Slomski, G. Landolt, S. Kittaka, Y. Maeno, J. H. Dil, I. S. Elfimov, M. W. Haverkort, and A. Damascelli, Phys. Rev. Lett. 112, 127002 (2014).

[43] Q. Han and A. Millis, Phys. Rev. Lett. 121, 067601 (2018).

[44] D. Sutter, C. Fatuzzo, S. Moser, M. Kim, R. Fittipaldi, A. Vecchione, V. Granata, Y. Sassa, F. Cossalter, G. Gatti, M. Grioni, H. M. Rønnow, N. C. Plumb, C. E. Matt, M. Shi, M. Hoesch, T. K. Kim, T. R. Chang, H. T. Jeng, C. Jozwiak, A. Bostwick, E. Rotenberg, A. Georges, T. Neupert, and J. Chang, Nat. Commun. 8, 15176 (2017).

[45] D. G. Porter, V. Granata, F. Forte, S. Di Matteo, M. Cuoco, R. Fittipaldi, A. Vecchione, and A. Bombardi, Phys. Rev. B 98, 125142 (2018).

[46] Y. Wang, J. J. Wang, J. E. Saal, S. L. Shang, L.-Q. Chen, and Z.-K. Liu, Phys. Rev. B 82, 172503 (2010).

[47] M. P. M. Dean, G. Dellea, R. S. Springell, F. Yakhou-Harris, K. Kummer, N. B. Brookes, X. Liu, Y.-J. Sun, J. Strle, T. Schmitt, L. Braicovich, G. Ghiringhelli, I. Božović, and J. P. Hill, Nat. Mater. 12, 1019 (2013).

[48] C. Monney, T. Schmitt, C. E. Matt, J. Mesot, V. N. Strocov, O. J. Lipscombe, S. M. Hayden, and J. Chang, Phys. Rev. B 93, 075103 (2016).

[49] J. Pelliciari, K. Ishii, Y. Huang, M. Dantz, X. Lu, P. OlaldeVelasco, V. N. Strocov, S. Kasahara, L. Xing, X. Wang, C. Jin, Y. Matsuda, T. Shibauchi, T. Das, and T. Schmitt, Commun. Phys. 2, 139 (2019).

[50] F. Forte, M. Cuoco, and C. Noce, Phys. Rev. B 82, 155104 (2010).

[51] M. Cuoco, F. Forte, and C. Noce, Phys. Rev. B 74, 195124 (2006).

[52] M. Cuoco, F. Forte, and C. Noce, Phys. Rev. B 73, 094428 (2006).

[53] J. C. Slater and G. F. Koster, Phys. Rev. 94, 1498 (1954).

[54] W. Brzezicki, C. Noce, A. Romano, and M. Cuoco, Phys. Rev. Lett. 114, 247002 (2015). 